\title{
DELIGNE HOMOLOGY AND ABEL-JACOBI MAPS
}

\author{
BY HENRI GILLET
}

The purpose of this note is to announce a functorial description of the Abel-Jacobi homomorphisms of [7] by means of a theory of cycle classes taking values in Deligne homology, which is part of a Poincaré duality theory satisfying the axioms of [3] on the category of all schemes of finite type over C. One consequence of this approach is that the cycle class map is an edge homomorphism in the coniveau spectral sequence, and so conjectures on the structure of the Chow groups, such as Bloch's conjecture [2], can be interpreted in terms of the vanishing (or not) of differentials in this spectral sequence. This formalism, because of its distinction between homology and cohomology, may also give a good framework for studying specialization questions. Deligne cohomology was originally defined by Deligne for $X$ a proper smooth variety (or manifold) over C:

$$
H^{p}(X, D(q))=\mathbf{H}^{p}\left(X^{a n}, \mathbf{Z}^{(2 \pi i)^{q}} \rightarrow O_{X} \rightarrow \cdots \rightarrow \Omega_{X}^{q-1}\right)
$$

so there are exact sequences for $p \geq 0$ :

$$
0 \rightarrow J^{p}(X) \rightarrow H^{2 p}(X, D(p)) \rightarrow H g e^{p}(X) \rightarrow 0
$$

( $J^{p}=p$ th Griffith's intermediate Jacobian [7]; $\mathrm{Hge}^{p}(X)$ is the group of integral $(p, p)$ cohomology classes). A description of these groups may be found in [5] together with a construction of cycle classes in them. The extension of the theory to noncompact, possibly singular, varieties, or even simplicial schemes, was obtained independently (and probably first) by A. A. Beilinson [1]. The definition of Deligne homology was inspired by El-Zein's and Zucker's use of currents to prove the covariant functoriality of the Abel-Jacobi maps in [5]. J. King has also proved naturality of the Abel-Jacobi maps by more explicit methods [8]. However, neither [5] nor [8] describe theories with the full range of formal properties described here.

Let $\mathcal{V}$ be the category of schemes of finite type over $\mathbf{C}, \mathcal{V}^{*}$ the category of closed immersions $Y \rightarrow X$ in $\mathcal{V}$ with morphisms Cartesian squares as in [3], and $\mathcal{V}_{*}$ the category of proper morphisms in $\mathcal{V}$.

THEOREM 1. There is a Poincaré duality theory with supports on $\mathcal{V}$, i.e., a pair of functors taking values in the category of bigraded abelian groups, one contravariant on $\mathcal{V}^{*}$,

$$
(Y \rightarrow X) \rightarrow \bigoplus_{p, q} H_{Y}^{p}(X, D(q))
$$

Received by the editors September 30, 1983.

1980 Mathematics Subject Classification. Primary 14C30, 14 C15.

(C) 1984 American Mathematical Society $0273-0979 / 84 \$ 1.00+\$ .25$ per page 
the other covariant on $\mathcal{V}_{*}$,

$$
X \rightarrow \bigoplus_{p, q} H_{p}(X, D(q))
$$

equipped with cap products

$$
H_{Y}^{p}(X, D(q)) \otimes H_{p}(X, D(s)) \rightarrow H_{r-p}(Y, D(s-q)) .
$$

These functors satisfy the axioms of [3], and, in addition, have the properties:

(1) If $X$ is smooth and proper over $\mathbf{C}$, then $H^{*}(X, D(*))$ are the groups defined by Deligne.

(2) There are long exact sequences

$$
\begin{aligned}
& \rightarrow \frac{H_{Y}^{p-1}(X, \mathbf{C})}{F^{q} H_{Y}^{p-1}(X, \mathbf{C})} \rightarrow H_{Y}^{p}(X, D(q)) \rightarrow H_{Y}^{p}(X, \mathbf{Z}(q)) \rightarrow \\
& \rightarrow \frac{H_{r+1}(X, \mathbf{C})}{F^{-s} H_{r+1}(X, \mathbf{C})} \rightarrow H_{r}(X, D(s)) \rightarrow H_{r}(X, \mathbf{Z}(s)) \rightarrow
\end{aligned}
$$

which are natural in $(Y \rightarrow X) \in\left(\mathcal{V}^{*}\right)$ and $X \in\left(\mathcal{V}_{*}\right)$.

(3) If $X$ is irreducible of dimension $d$, there is a canonical class $\eta(Y) \in$ $H_{2 d}(X, D(d))$ such that

$$
H_{2 d}(X, D(d)) \simeq \mathbf{Z} \eta(Y) \text {. }
$$

(4) There are cup products for $(Y \rightarrow X),(Z \rightarrow X)$ in $\mathcal{V}^{*}$,

$$
H_{Y}^{p}(X, D(q)) \otimes H_{Z}^{r}(X, D(s)) \rightarrow H_{Y \cap Z}^{p+r}(X, D(q+s)),
$$

compatible with the cap products.

COROLlaRY. There are short sequences for $X \in \mathcal{V}$,

$$
0 \rightarrow J^{p}(X) \rightarrow H^{2 p}(X, D(p)) \rightarrow H g e^{p}(X) \rightarrow 0
$$

and

$$
0 \rightarrow J_{p}(X) \rightarrow H_{2 p}(X, D(p)) \rightarrow H_{2 p}(X, \mathbf{Z}(p)) \cap F^{-p} H_{2 p}(X, \mathbf{C}) \rightarrow 0,
$$

where $J^{p}$ and $J_{p}$ are the cohomology and homology versions of Griffith's intermediate Jacobians $\left(H^{2 p}(X, D(p)) \stackrel{\text { dof }}{=} H_{X}^{2 p}(X, D(p))\right)$.

TheOREM 2. (i) For $X \in \mathrm{Ob}(\mathcal{V})$ and $n \in \mathbf{Z}$, there are spectral sequences

$$
\begin{aligned}
& E_{p, q}^{1}(X, n)=\bigoplus_{x \in X_{(p)}} H_{p+q}(x, D(n)) \Rightarrow H_{p+q}(X, D(n)), \\
& E_{1}^{p, q}(X, n)=\bigoplus_{x \in X^{(p)}} H^{q-p}(x, D(n)) \Rightarrow H^{q-p}(X, D(n))
\end{aligned}
$$

$\left(X^{(p)}, X_{(p)}\right.$ are the sets of points of codimension and dimension $p$ in $X$, respectively) such that $E_{p, p}^{2}(X, p)=C X_{p}(X)$ and $E_{2}^{p, p}(X, p)=C H^{p}(X)$ (the Chow groups of $X[6])$. The edge homomorphisms $E_{p, p}^{2}(X, p) \rightarrow H_{2 p}(X, D(p))$ therefore define cycle class maps $C H_{p}(X) \rightarrow H_{2 p}(X, D(p))$ which are covariant for proper maps. By duality, if $Y$ is codimension $p$ on a smooth $X$, there is a cycle class $\eta(Y) \in H_{Y}^{2 p}(X, D(p))$. 
(ii) If $X$ is smooth there are isomorphisms

$$
E_{2}^{p, q}(X, n) \simeq H^{p}\left(X, \nvdash^{q}(D(n))\right),
$$

where $\mathcal{H}^{q}(D(n))$ is the Zariski sheaf associated to Deligne cohomology. In particular:

$$
C H^{p}(X) \simeq H^{p}\left(X, H^{p}(D(p))\right)
$$

(iii) If $X$ is proper and smooth, the cycle class map of (i), when restricted to cycles homologically equivalent to zero $\left(C H^{*}(X)_{\sim 0}\right)$ gives a map, for $p \leq 0$,

$$
C H_{p}(X)_{\sim 0} \rightarrow J_{p}(X)
$$

which agrees with the Abel-Jacobi map of [7] and which, by (i), is a natural transformation of covariant functors.

(iv) The cycle class maps, for $X$ smooth not necessarily compact;

$$
\eta: C H^{*}(X) \rightarrow \bigoplus_{p} H^{2 p}(X, D(p))
$$

form a natural transformation of contravariant ring-valued functors. In particular, the Abel-Jacobi maps $C H^{p}(X)_{\sim 0} \rightarrow J^{p}(X)$ are compatible with pullbacks.

Corollary. For $X$ a smooth projective surface over $\mathbf{C}$ there is an exact sequence coming from $E_{2}(X, 2)$ :

$$
H^{3}(X, D(2)) \rightarrow H^{0}\left(X, \not H^{3}(D(2))\right) \stackrel{d_{2}^{0,3}}{\rightarrow} C H^{2}(X) \sim 0 \stackrel{\eta}{\rightarrow} J^{2}(X) \rightarrow 0 .
$$

Hence the obstruction to $\eta$ being an isomorphism (as conjectured in [2] when $\left.p_{g}(X)=0\right)$ is the nonvanishing of the differential $d_{2}^{0,3}$.

Sketch of Proofs. If $u: Z$. $\rightarrow \bar{Z}$. is a normal crossings compactification of a smooth simplicial scheme and $Y .=\bar{Z}$. $-Z$., set (see [4] for the notation regarding forms with logarithmic poles)

$$
D_{\bar{Z} .}\langle Y .\rangle(s)=C\left(R u_{*} \mathbf{Z} \oplus \Omega_{\bar{Z}}<^{<s}\langle Y .\rangle^{(2 \pi i)^{s}+u^{*}} \rightarrow u_{*} \Omega_{Z}\right) \quad[\mathbf{1}]
$$

where $R u_{*}$ is computed using Godement resolutions. Then define $H^{p}(Z,, D(s))$ to be $\mathbf{H}^{p}\left(\bar{Z} ., D_{\bar{Z}}\langle(Y).(s))\right.$. For $X$ in $\mathcal{V}, H^{p}(X, D(q))$ is defined as $H^{p}(Z ., D(q))$ for $Z$. a proper smooth hypercover of $X$, while $H_{Y}^{p}(X, D(q))$ is constructed using the mapping cone of a map of hypercovers covering the inclusion $(X-$ $Y) \rightarrow X$. If $X$ is proper and smooth, Deligne homology is defined using currents as in [5]. For $X$ proper and possibly singular, we take the double complex of currents associated to a hypercover of $X$, and for $X$ nonproper, $X \rightarrow \bar{X}$ a compactification, we take $H_{p}(X, D(q))=H_{p}(\bar{X}, \bar{X}-X ; D(q))$. Cap products are defined using residues. Theorems 1 and 2 then follow using the techniques of [ 3 and 4], except that contravariant functoriality is proved as it is for most theories of cycle classes by reducing to the case of intersections with Cartier divisors. 


\section{REFERENCES}

1. A. A. Beilinson, Higher regulators and values of $L$-functions, 1983, (manuscript).

2. S. Bloch, $K_{2}$ of Artinian Q-algebras, with applications to algebraic cycles, Comm. Algebra 3 (1975), 405-428.

3. S. Bloch and A. Ogus, Gersten's conjecture and the homology of schemes, Ann. Sci. Ecole Norm. Sup. 7 (1974), 181-202.

4. P. Deligne, Theorie de Hodge. II, III, Inst. Hautes Études Sci. Publ. Math. 40 (1971), 5-77; ibid. 44 (1974), 5-77.

5. F. El-Zein and S. Zucker, Extendability of normal functions associated to algebraic cycles, 1982, (preprint).

6. W. Fulton, Intersection theory, Ergebnisse Math. Grenzgeb., 3 Folge, vol. 2, SpringerVerlag, 1983.

7. P. Griffiths, Some results on algebraic cycles on algebraic manifolds, Algebraic Geometry, Tata/Oxford, 1969.

8. J. King, Log complexes of currents and functorial properties of the Abel-Jacobi map, Duke Math. J. 50 (1983), 1-54.

Department of Mathematics, Princeton University, Princeton, New JERSEY 08544

Current address: Department of Mathematics, University of Pennsylvania, Philadelphia, Pennsylvania 19104 